تشخيص عطب عدم إنتظام الفجوة الهوائية المختلط في المولا التزامني ذي الاقطاب

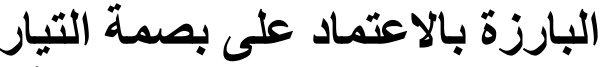

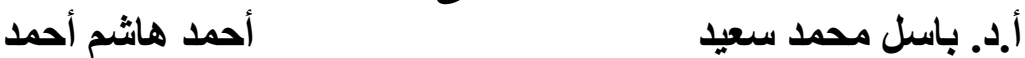

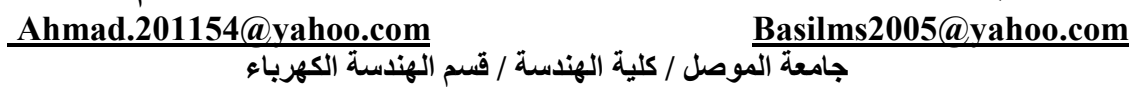

\title{
الخلاصة الفجوة الخوان
}

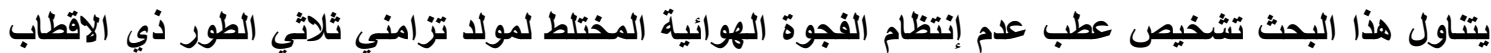

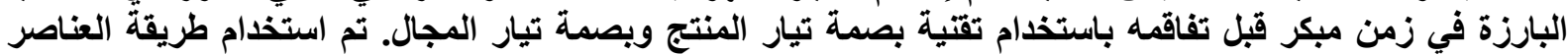

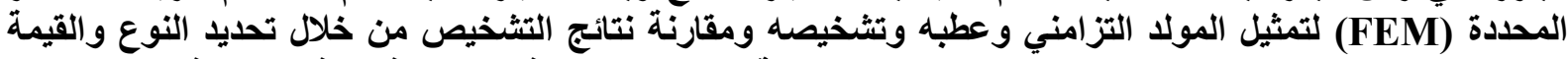

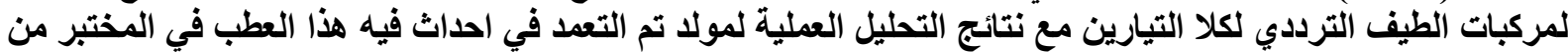

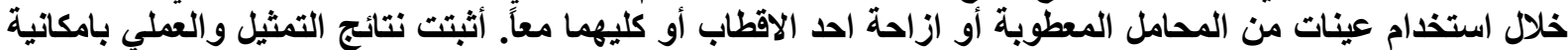

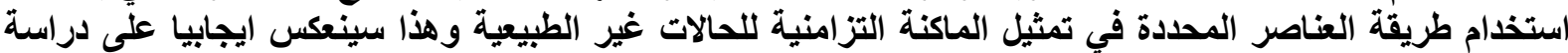
سلوك الماكنة عند حدوث عطب سبية كهربائي أو ميكانيكي. الكلمات المفتاحية: عدم إنتظام الفجوة الهوائية، طريقة عيلة العناصر المحددة، تحليل بصمة تيار.

\section{Mixed Air Gap Eccentricity Fault Diagnosis of a Salient Pole Synchronous Generator Based on Current Signature}

\author{
Prof. Dr.Basil M. Saied \\ Basilms2005@yahoo.com
}

\author{
Ahmed . H. Ahmed \\ Ahmad.201154@yahoo.com
}

/ Mosul university Electrical Eng. Dept.

\begin{abstract}
This paper presents an air gap eccentricity fault Diagnosis of a salient Pole three phase synchronous generator. The detection and diagnosis of this type of fault is based on analyzing armature and field current signatures, using FFT . Therefore, the synchronous generator, under this type of fault, is modeled by using finite element method (FEM). Also practical results are performed in the lab with deliberately air gap eccentricity faults, by using samples of bearings malfunctioning or the removal of single or pair pole. The modeling and experimental results show that FEM is a reliable approach that can be used to model synchronous generator under electrical and mechanical faults.
\end{abstract}

Keywords: Air Gap Eccentricity, Finite Element Method, Current Signature. 


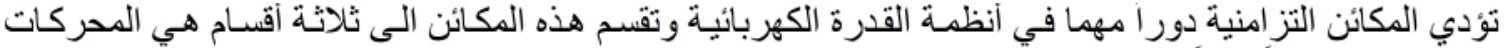

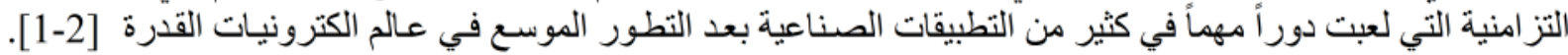

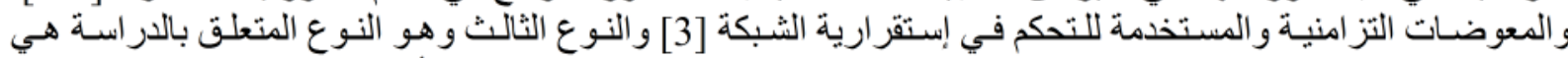

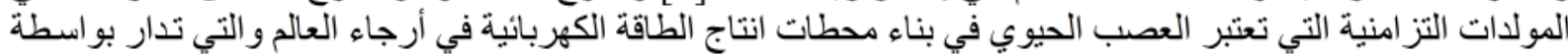

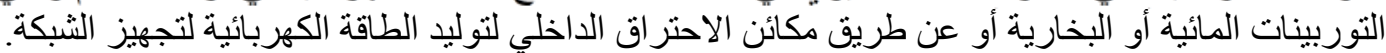

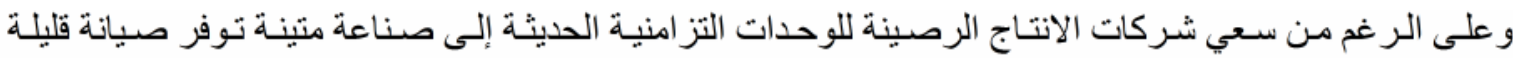

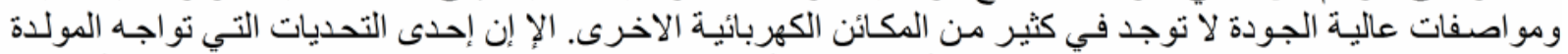

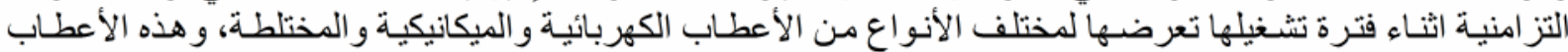

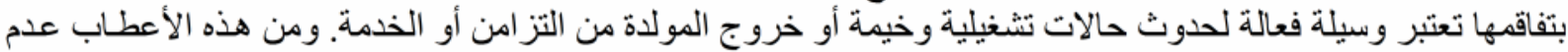

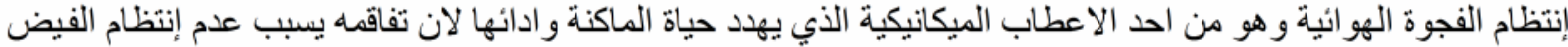

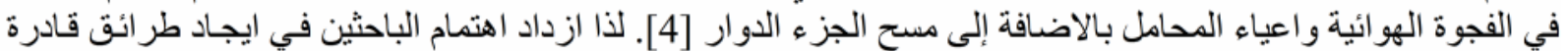

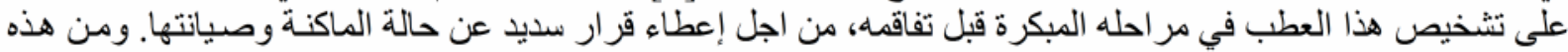

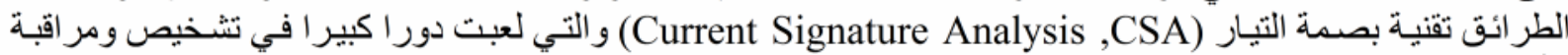

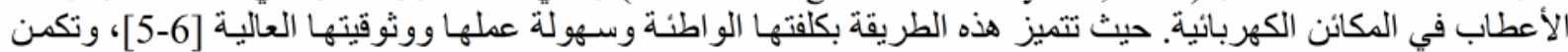

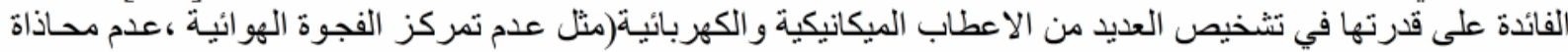

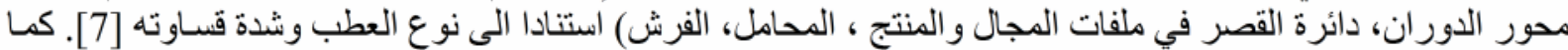

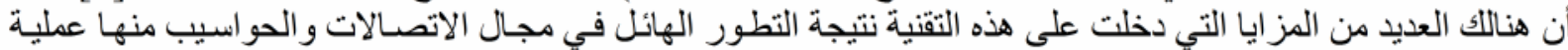

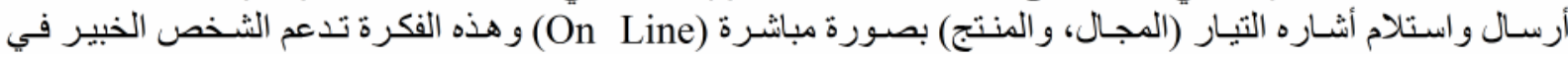

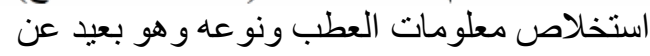

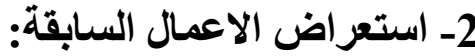

تطرق كثير مِن البـاحثين إلى تشخيص لعطب عدم إنتظـام الفجوة الهو ائية في الماكنة التز امنيـة وتنو عت ابحاثهم

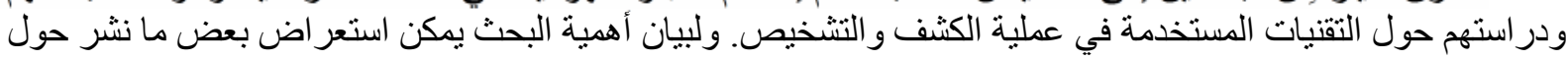
هذا الموضوع.

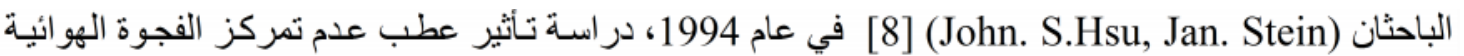

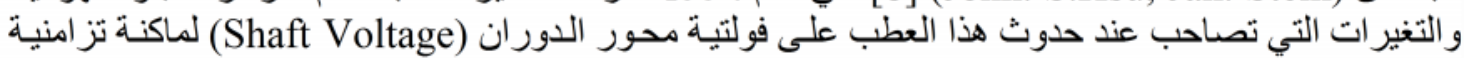

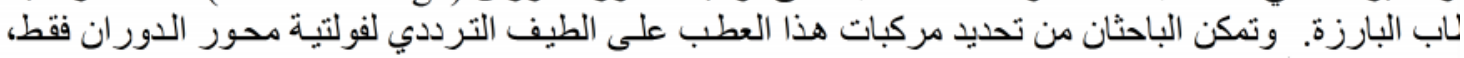

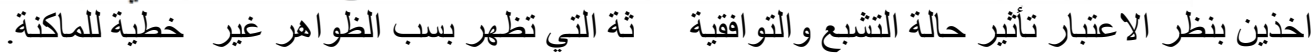

(I. Tabatabaei)

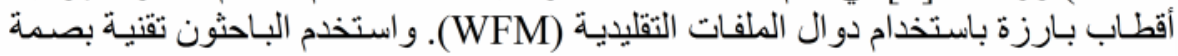
تيار المنتج ومر اقبة التغير الحاصل في المحاثة التبادلية بين الاطوار الجزء الباء الساكن لكشف وتثخيص هذا العطب.

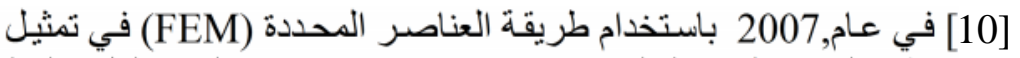

(M. Kiani)

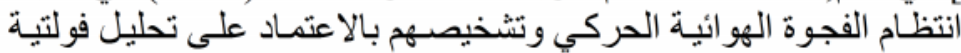

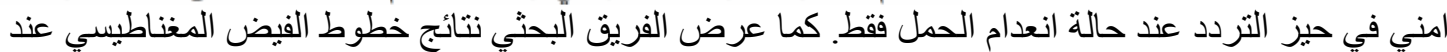

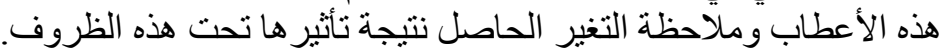

وفريقه البحثي [11] في عام 2009، باستخدام تقنية تحليل فولتية المنتج في حيز

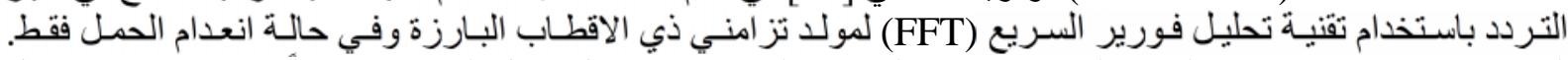

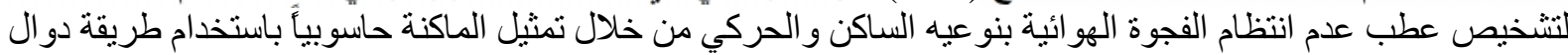

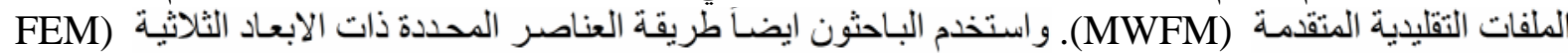




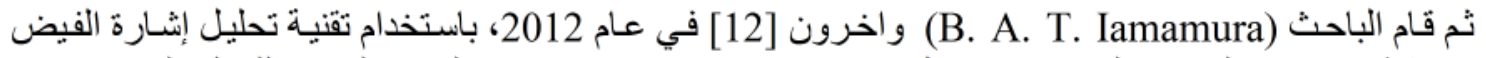

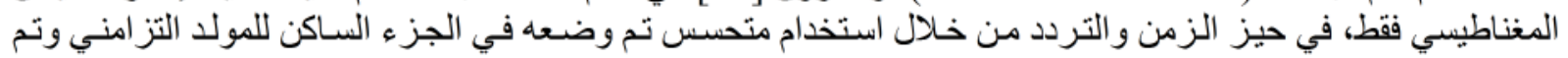

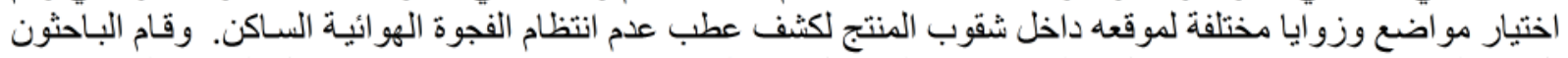

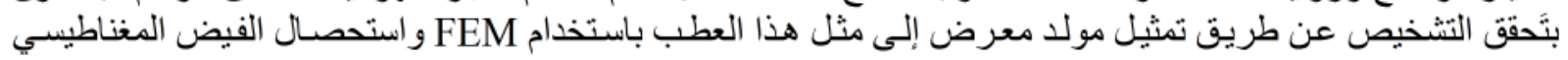
المطلوب لمقارنته مع الفيض القادم من المتحس.

\section{Air Gap Eccentricity}

\section{3-عطب عدم إنتظام الفجوة الهوائية}

تمركز الفجوة الهوائية هو احد الاعطاب الميكانيكية التي يمكن للمولدة التزامنية ذات

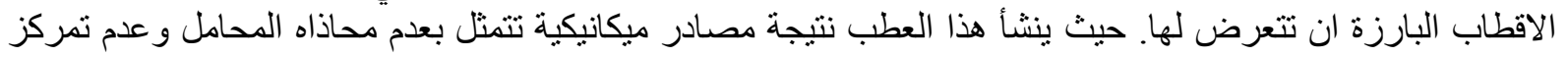

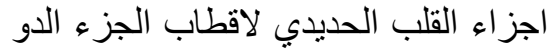

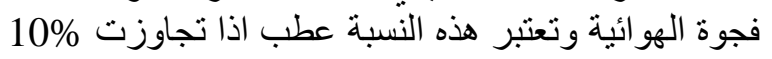

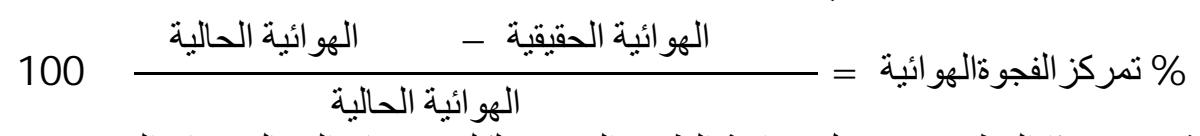

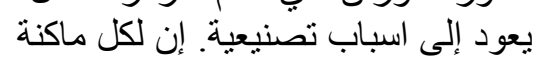

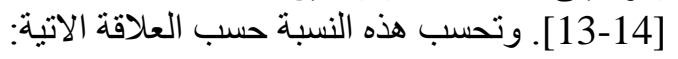

يمكن كثف وتتخيص هذا العطب من خلال مر اقبة الطيف الترددي لكل من تيار المجال وتيار المنتج (بصمة التئية التبار )

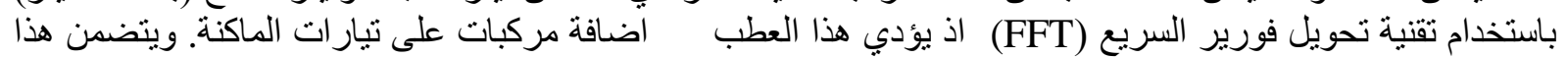

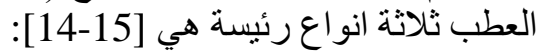

\section{Static Air Gap Eccentricity}

(Oc)

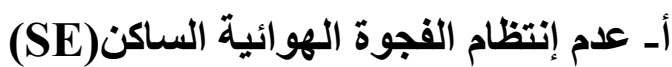

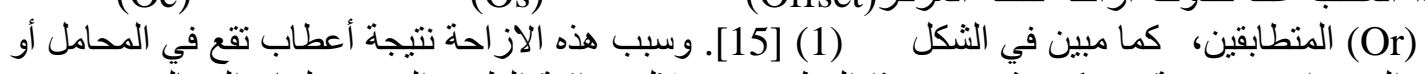

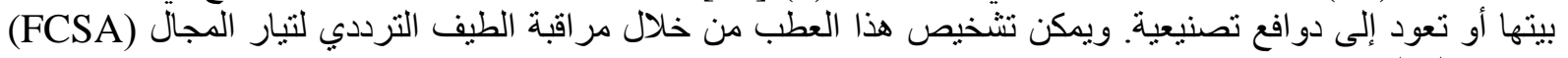
$F_{F S E}=\left(6 h \pm \frac{k m}{p}\right) f S$

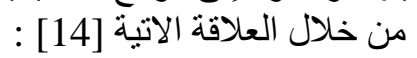

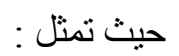

FSE

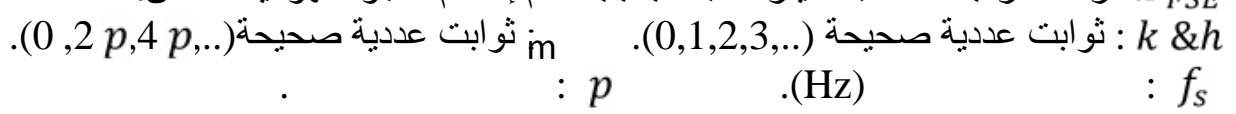
كما يمكن مر اقبة الطيف الترددي لتبار المنتج (ACSA) من خلال العلاقة الاتية:

$F_{S S E}=\left(n \pm 6 h \pm \frac{k m}{p}\right) f S$

حيث تمثل:

FSE تردد المركبات الناتجة لتبار المنتج بسبب عدم إنتظام الفجوة الهو ائية الساكن (Hz). n : ثو ابت عددية صحيحة (......

\section{Dynamic Air Gap Eccentricity}

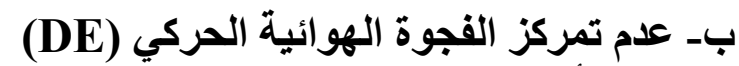

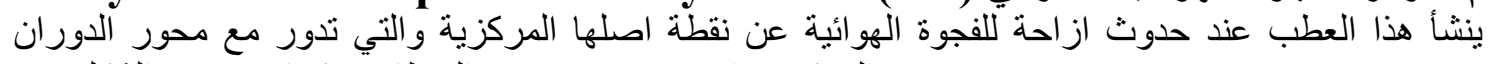

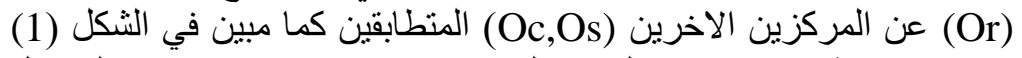

(Shaft)

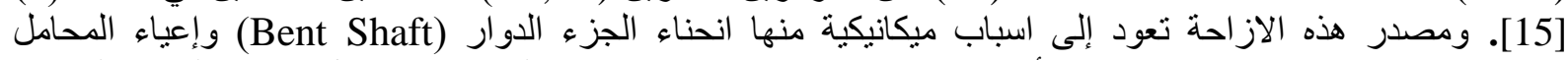

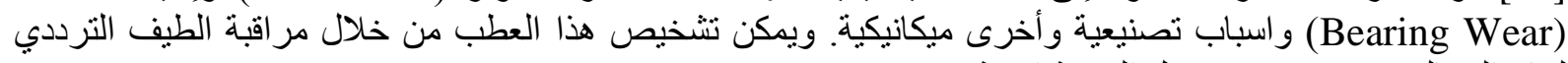

$$
F_{F D E}=\left(6 h \pm \frac{k m}{p}\right) f s \quad m=0 ;
$$

التيار المجال (FCSA) من خلال العلاقة الاتية [14] : 
:FDE

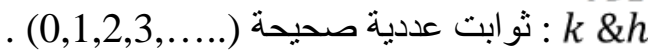
كما يمكن مر اقبة الطيف الترددي لتيار المنتج (ACSA) من خلال العلاقة الاتية:

$F_{S D E}=\left(n \pm 6 h \pm \frac{k m}{p}\right) f s \quad m=0$;

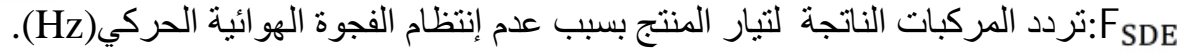

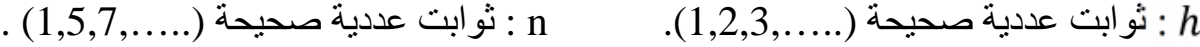

\section{Mixed Air Gap Eccentricity}

(Os)
ج- عدم تمركز الفجوة الهوائية المختلط (ME)

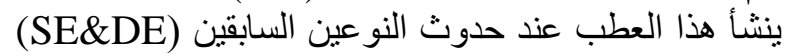

(Or)

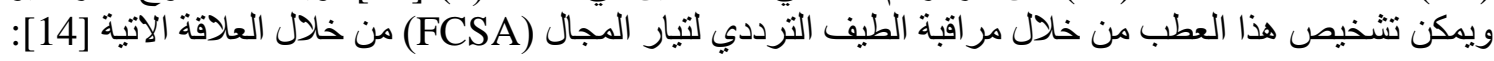

$$
F_{F M E}=\left(6 h \pm \frac{k m}{p}\right) f s
$$

$$
\begin{aligned}
& \text { FFE تردد المركبات الناتجة لتيار المجال بسبب عدم إنتظام الفجوة الهو ائية المختلط بوحدة (Hz). }
\end{aligned}
$$

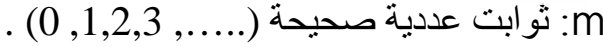

$$
\begin{aligned}
& \text { : ثو ابت عددية صحيحة (0.... k \&h }
\end{aligned}
$$

كما يمكن مر اقبة الطيف الترددي لتيار المنتج (ACSA) من خلال العلاقة الاتية:

$$
F_{S M E}=\left(n \pm 6 h \pm \frac{k m}{p}\right) f s
$$

( $(\mathrm{Hz})$

إنرد

$$
\text { n : ثوابت عددية صحيحة (1, } 1,5,7, \ldots . .) .
$$

h : ثو ابت عددية صحيحة (....

m: ثوابت عددية صحيحة (....,

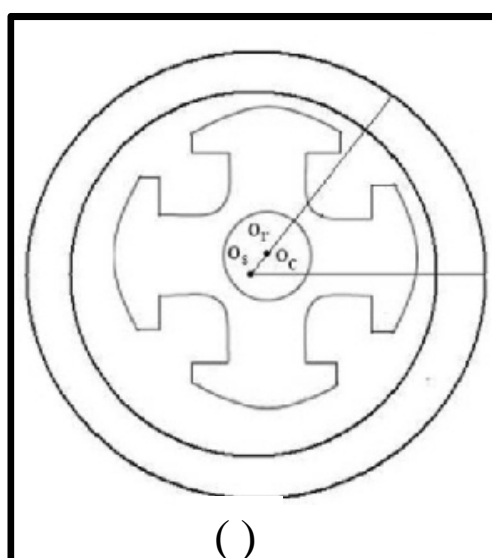

SE

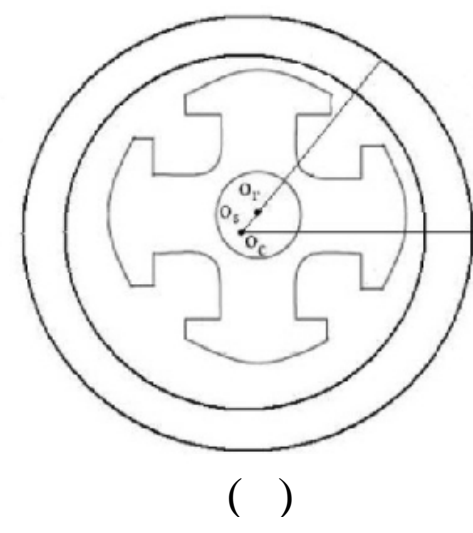

$\mathrm{DE}$

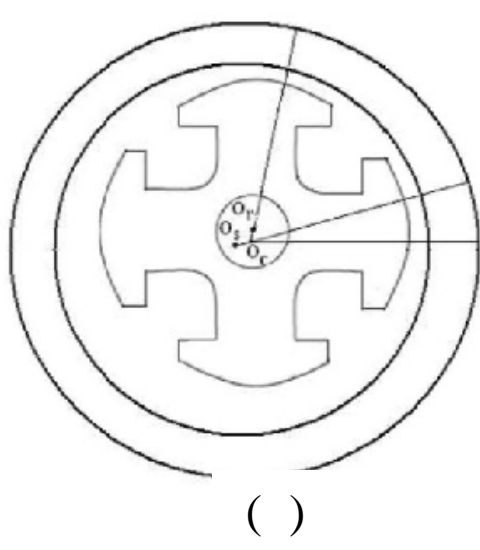

$\mathrm{ME}$

(1) الماكنة تحت عطب عدم تمركز الفجوة الهو ائية.

(أ) عدم تمركز الفجوة الهو ائية الساكن. (ب) عدم تمركز الفجوة الهوائية الحركي. (ج) عدم تمركز الفجوة الهوائية المختلط. 


\section{3- تمثيل عطب عدم إنتطام الفجوة الهوائية المختلط ونتائج تثخيصه:}

تم تمثيل عطب عدم إنتظام الفجوة الهوائية المختلط عن طريق ازاحة الجزء الدوار و نقطة تمركز محور دوران

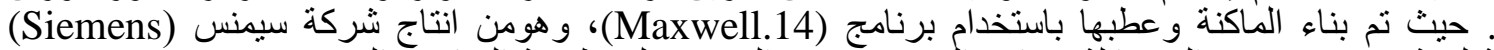

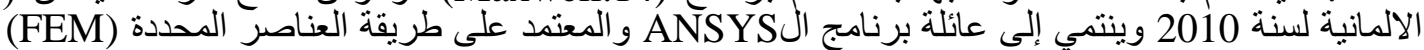

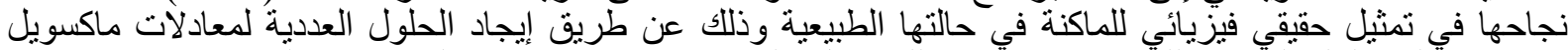

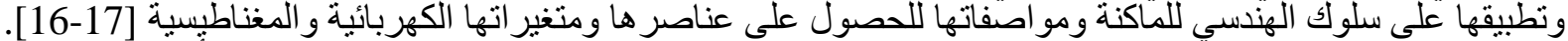

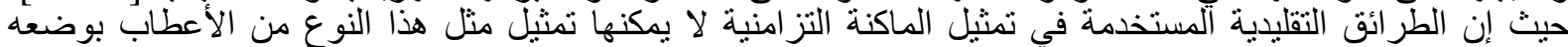

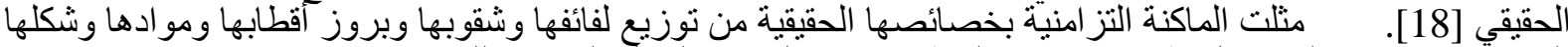

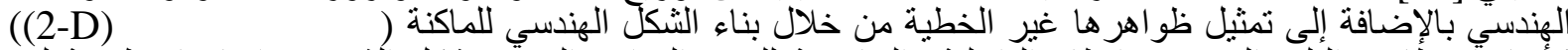

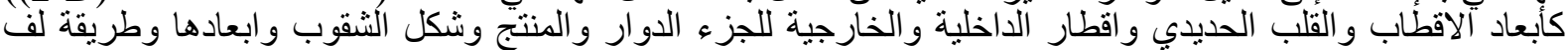

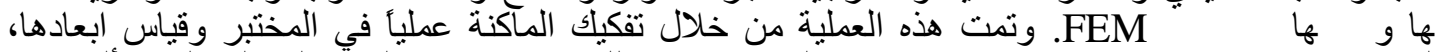

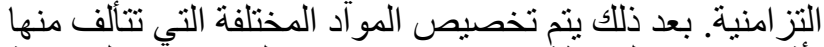

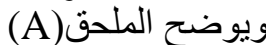

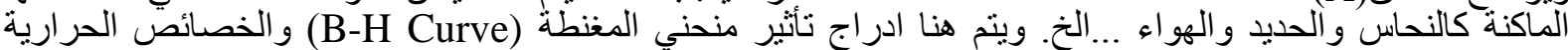

للمو اد الكهربائية والمغناطيسية.

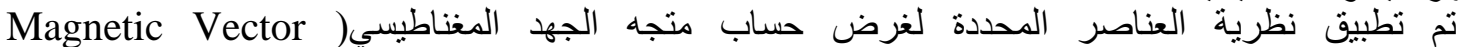

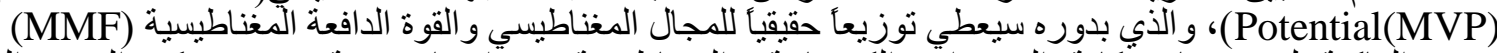

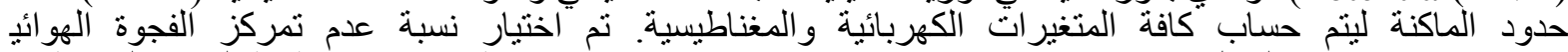

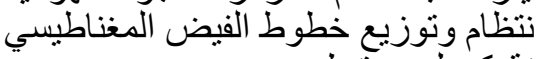

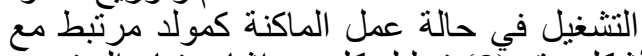

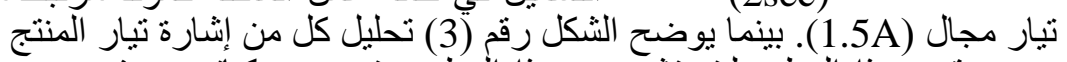

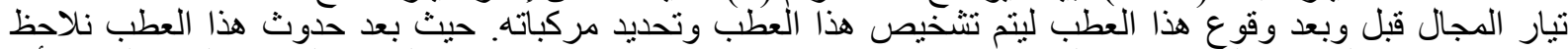

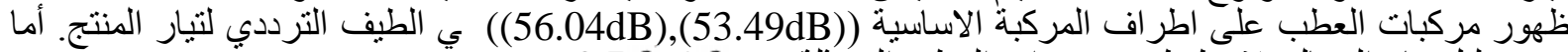

عند تحليل تيار المجال نلاحظظ ظهور ترددات العطب المتمثلة (0.5f $)$ (
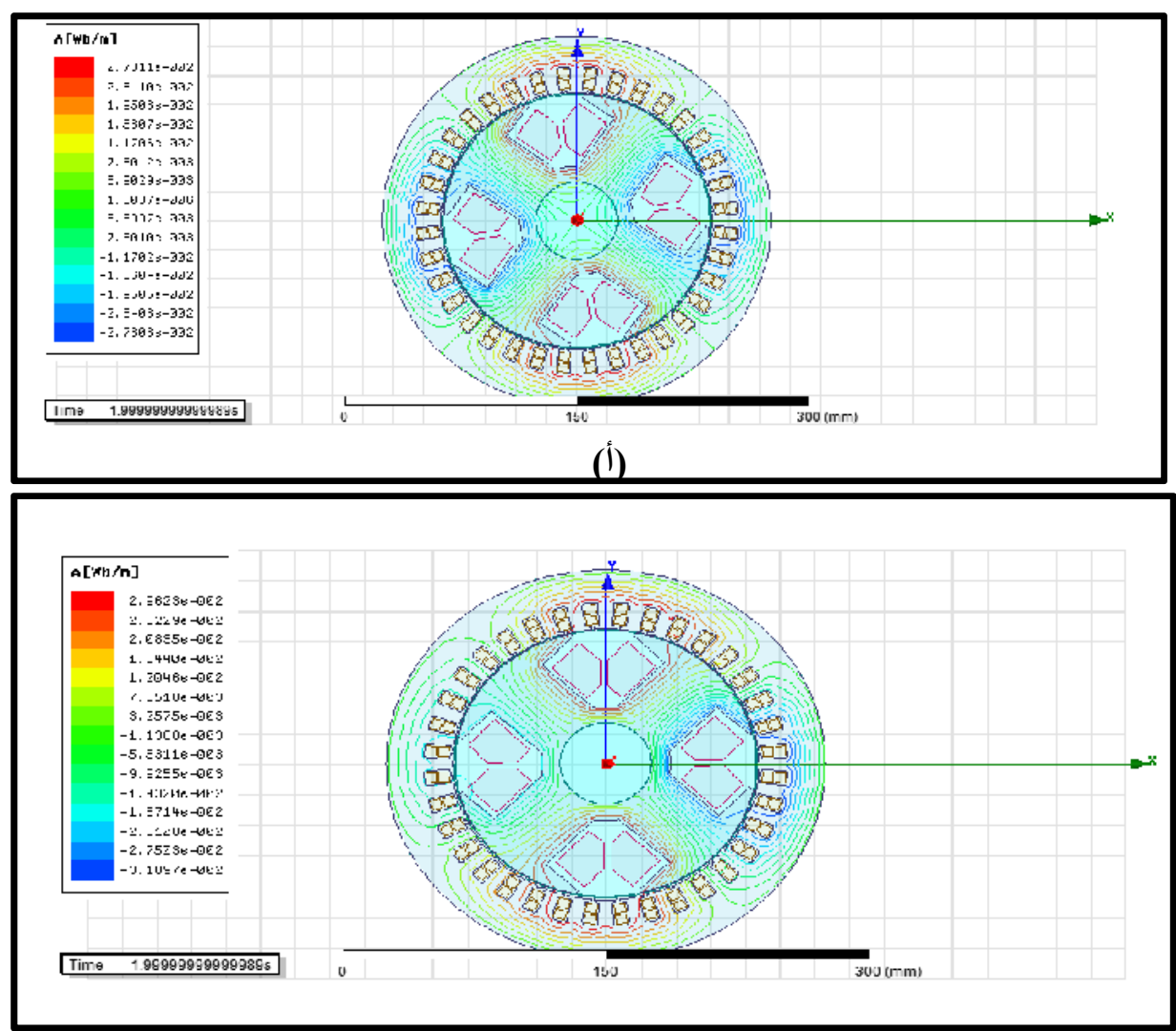

$(ب)$

وجود عدم إنتظام الفجوة الهو ائية المختلط.

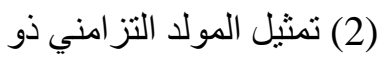

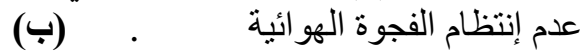

(ا) 

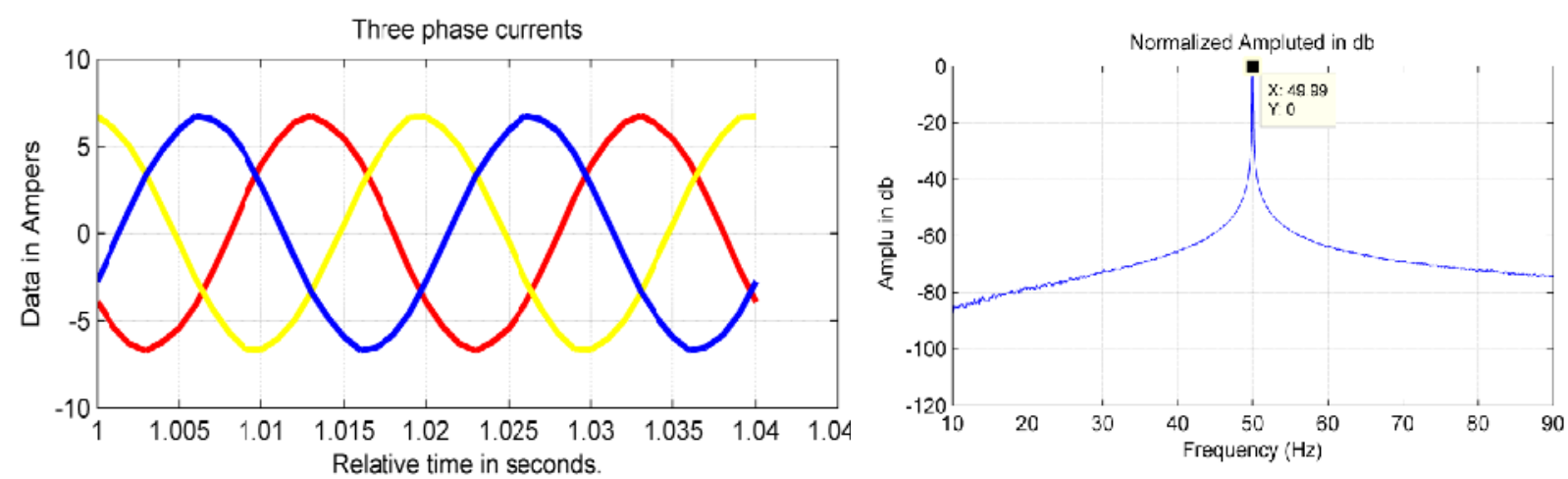

Field current
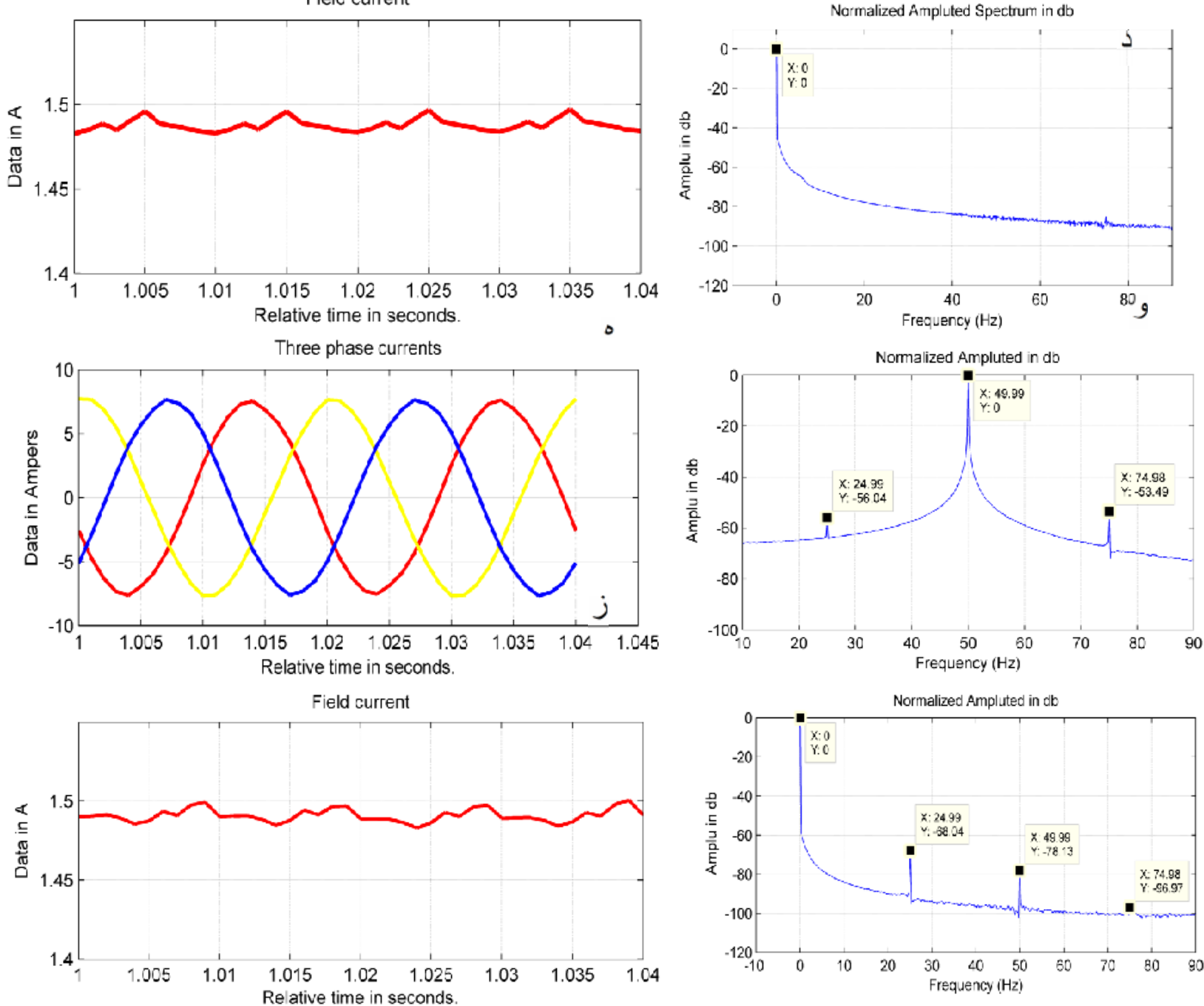

(3) نتائج التمثيل بالحاسبة لتيار المنتج وتيار المجال لمولد تز امني :

( ) إنشارة تيار المنتج في حيز الزمن قبل العطب. تحليل إثنارة تيار المنتج في الحيز الترددي قبل العطب. ( ) إثارة تيار المجال في حيز الزمن قبل العطب. ) تحليل إثارة تيار المجال في الحيز الترددي قبل بعطب. ( ) إنشارة تيار المنتج في حيز الزمن بعد العطب. تحليل إثنارة تيار المنتج في الحيز الترددي بعد العطب. ( ) إنشارة تيار المجال في حيز الزمن بعد العطب. تحليل إثنارة تيار المجال في الحيز الترددي بعد العطب. 
4- المنظومة العملية المستخدمة في الكثف والتشخيص:

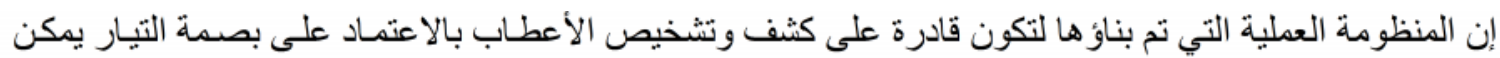

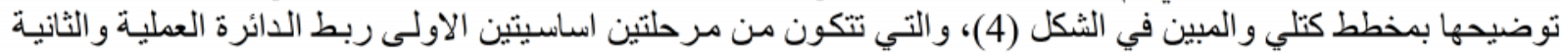

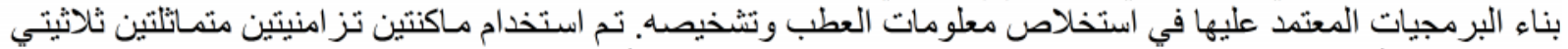

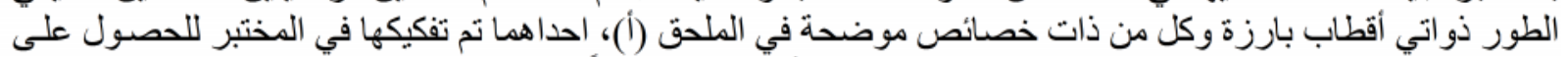

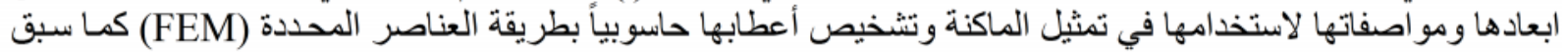

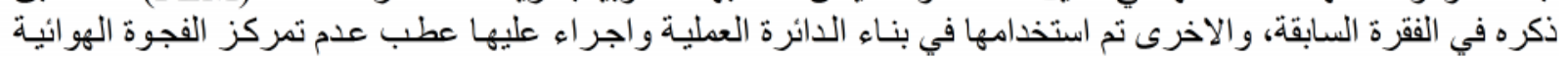

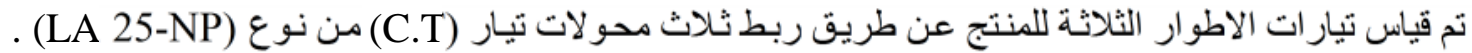

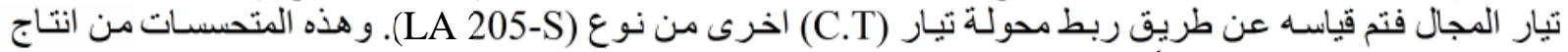

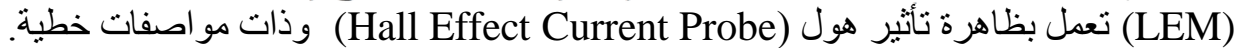
بعد تكيف الإشار ات وعزلها بوة بلاسطة المتحسسات تم ادخال هذه الإشارات إلى الحاسبة عن طريق بطاقة اكتساب (MATLAB) Advantech PCI 1710 البيانات البرة

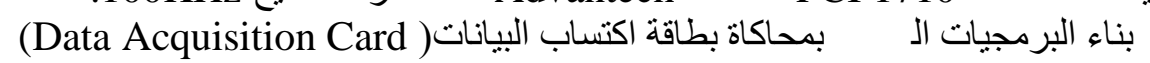

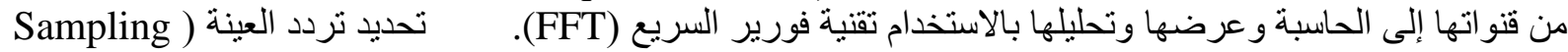
(Frequency

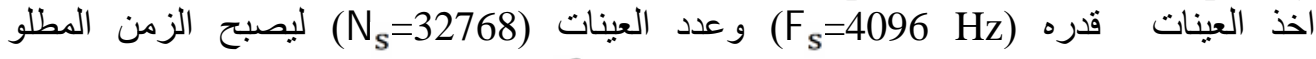

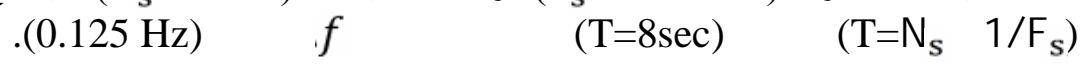

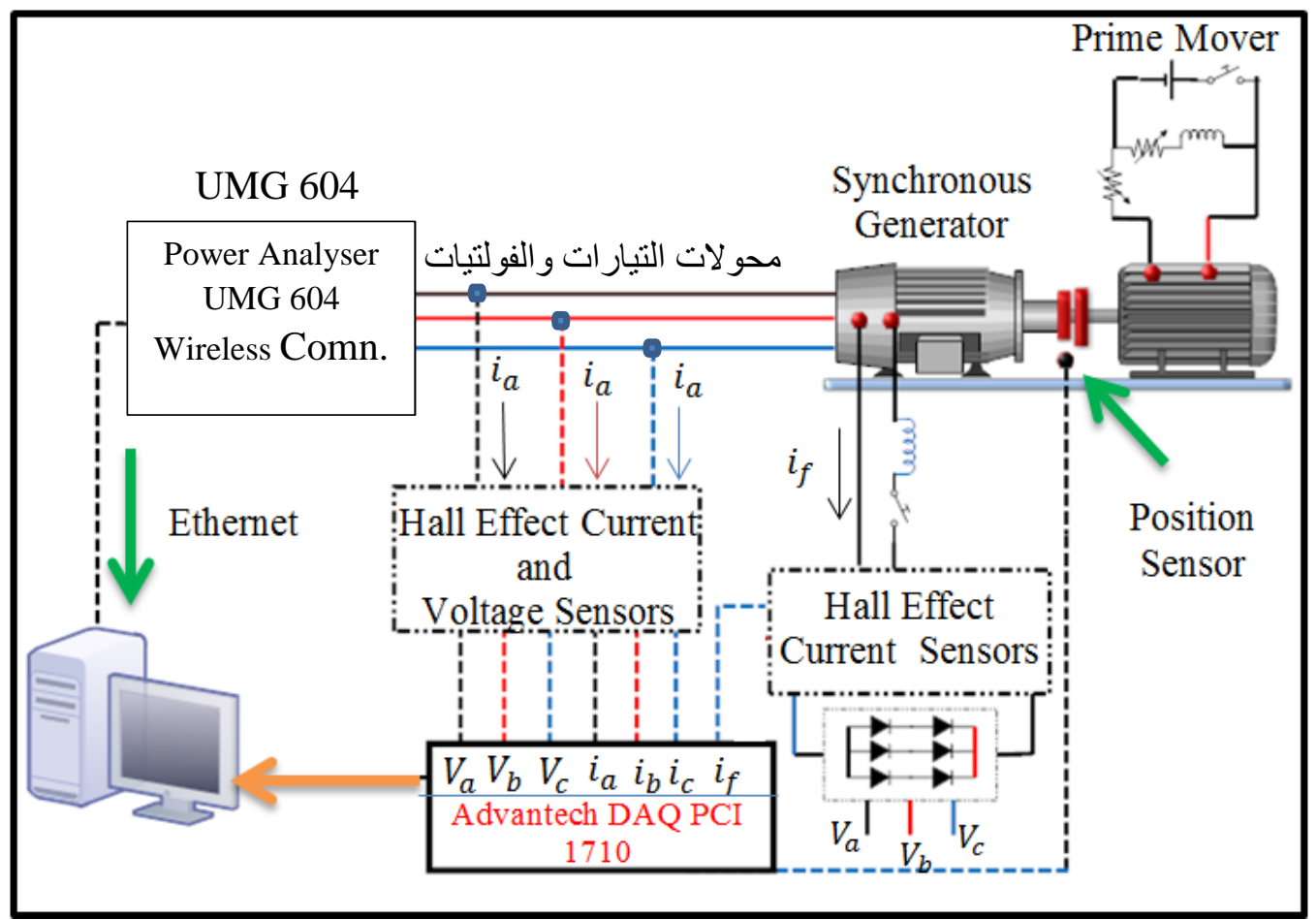

(4) المخطط الكتلي للمنظومة العملية المستخدمة في الكثف و التشخيص و التحليل.

5- محاكاة عطب عدم انتظام الفجوة الهوائية المختلط ونتائج تثخيصه:

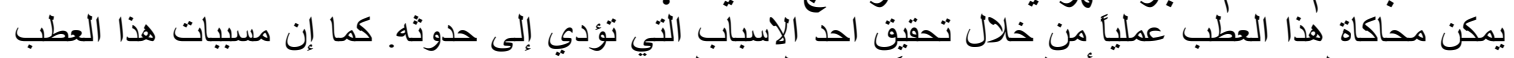

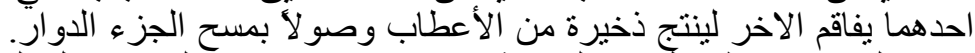

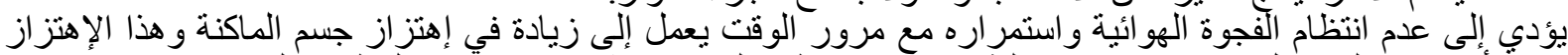

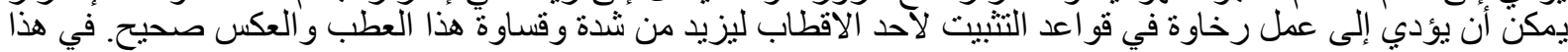




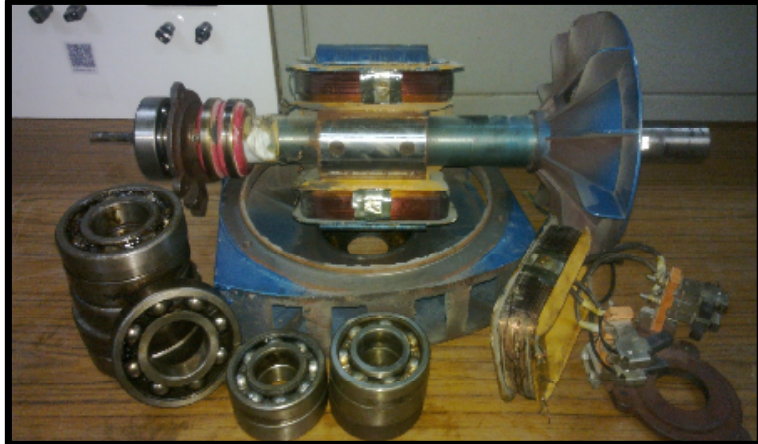

(5) محاكاة عطب عدم إنتظام الفجوة الهو ائية المختلط.
قيق هذا النوع من الأعطاب وريق استخدام

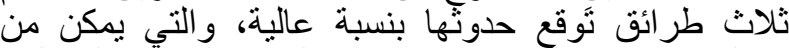

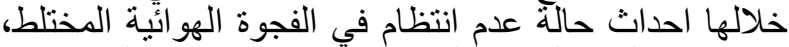
وبنسب مختلفة اعلى من المسموح بها. وكانت النهام هذه الطر ائق:2- استخدام عينات من المحامل المعطوبة والتي أدت إلى مدي حدوث مثل هذا العطب. 3- استخدام عينات المحامل وإز احة احد الحد الاقطاب في الوقت نفسه. الثكل (5) يوضن الثح محاكاة هذا العطب.

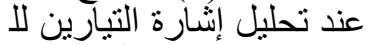
العطب، و عند تشغيل الماكنة

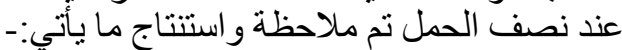

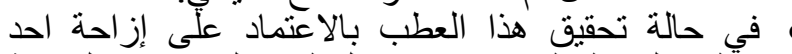

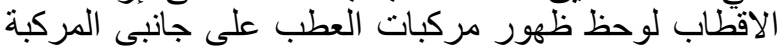

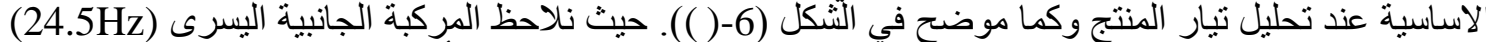
(42.7dB)

$$
\text { (()-6) }
$$

$(73.38 \mathrm{~Hz})$

$$
\text { (-62.42dB) (24.5Hz) }
$$

• في حالة تحقيق هذا العطب بالاعتماد على استخدام عينات المحامل المعطوبة لوحظ ظهور مركبات

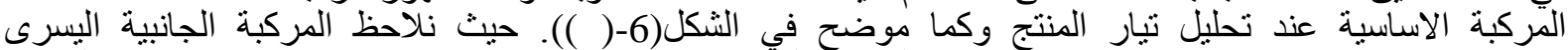
بيل تبار
. $(-58.19 \mathrm{~dB})$
(74.38Hz)

ل) (24.88Hz)

(24.75Hz)

.(( )-6)

• في حالة تحقيق هذا العطب بالاعتماد على الطريقتين السابقتين في الوقت نفسه لوحظ ظهور مركبات العطب على جانبي

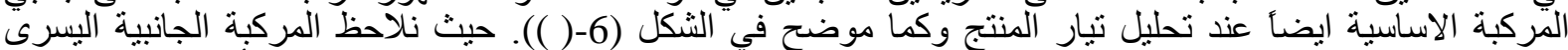

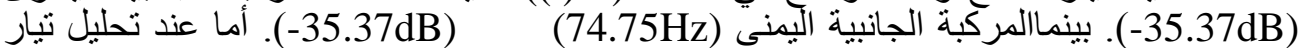
.(( )-6) . $(-49.99 \mathrm{~dB})$
$(24.88 \mathrm{~Hz})$

$(24.88 \mathrm{~Hz})$

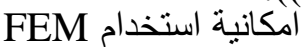

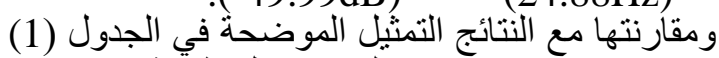

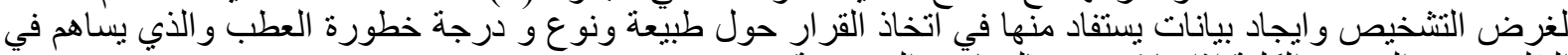

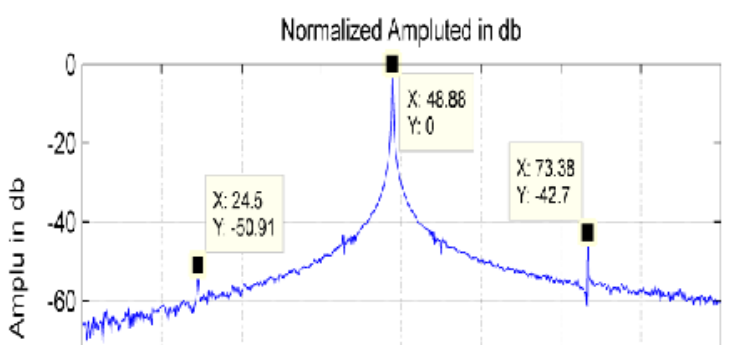

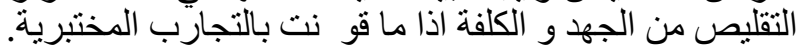

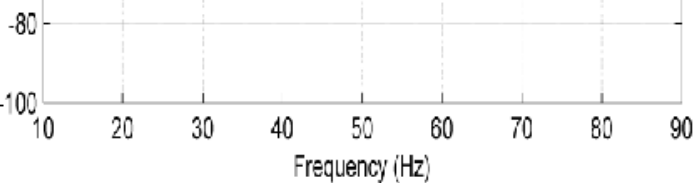

Normalized Amoluted in db
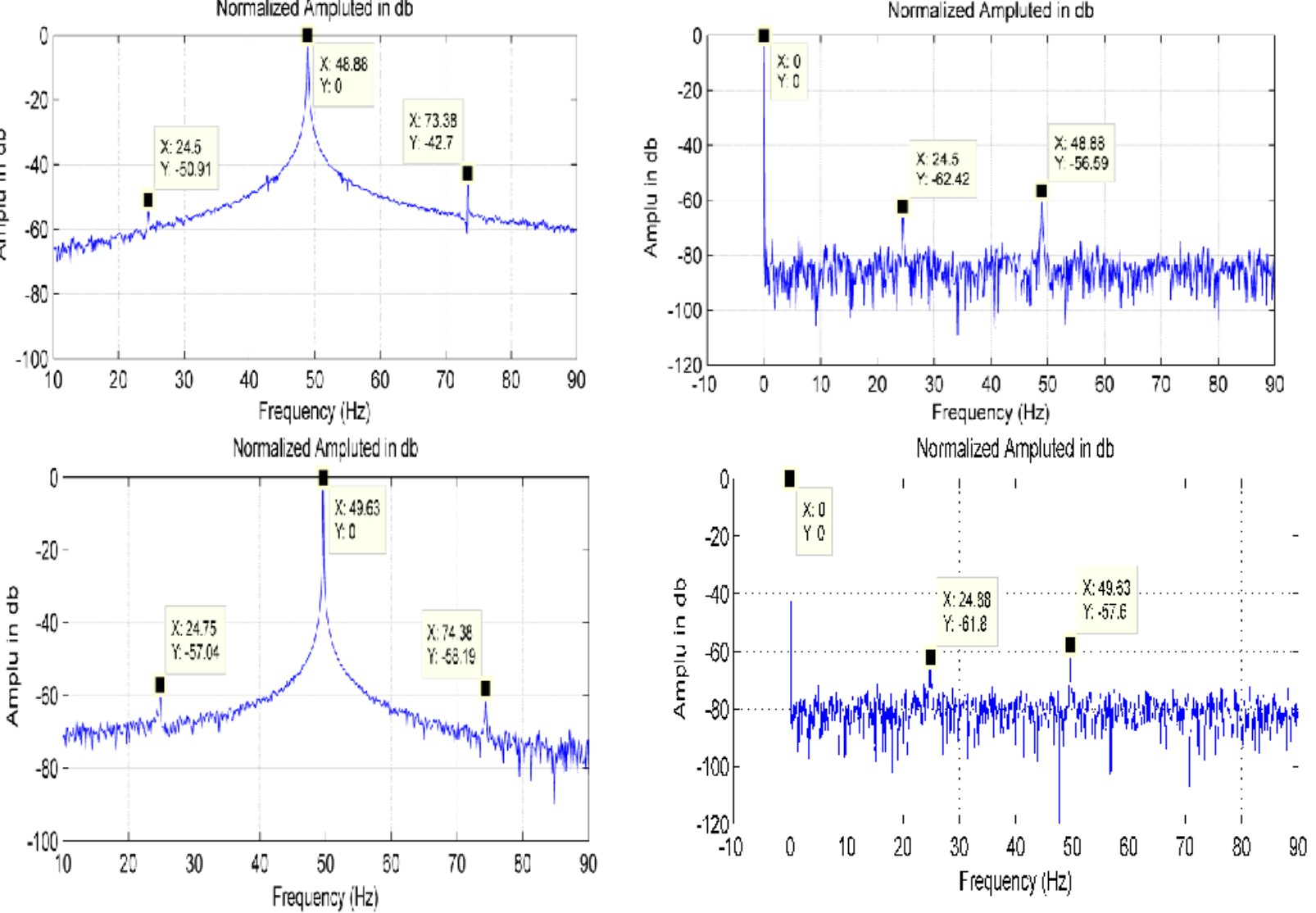

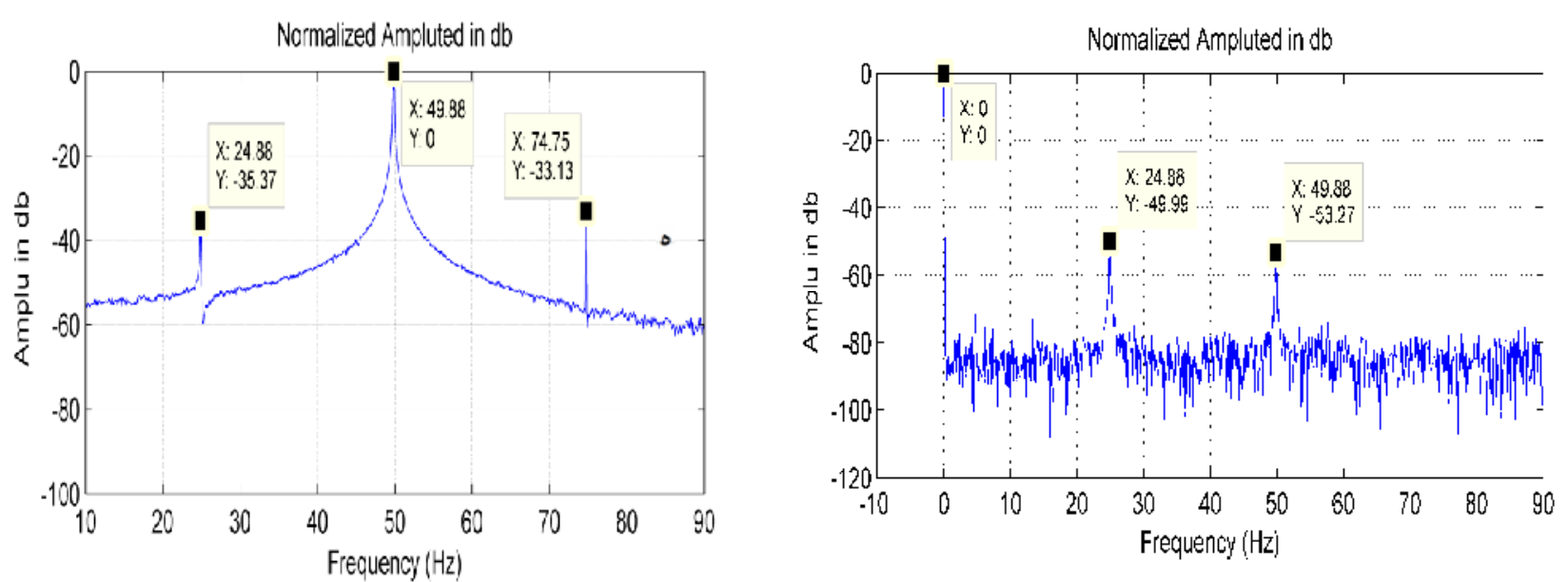

( ) تبار المنتج في حيز الترددي عند ازاحة احد الاقطاب. ( ) تبار المجال في الحيز الترددي عند

( ) تيار المنتج في حيز الترددي عند المحامل المعطوبة. ( ) تيار المجال في الحيز الترددي عند المحامل المعطوبة.

$$
\begin{aligned}
& \text { ( ) تيار المنتج في حيز الترددي عند از احة احد الاقطاب و المحامل المعطوبة معأ. } \\
& \text { ( ) تيار المجال في الحيز الترددي عند ازاحة احد }
\end{aligned}
$$

\begin{tabular}{|c|c|c|c|c|}
\hline \multicolumn{2}{|c|}{ تيار المجال (dB) } & \multicolumn{2}{|c|}{ تيار المنتج (dB) } & \\
\hline $1 f_{s}$ & $0.5 f_{s}$ & $1.5 f_{s}$ & $0.5 f_{s}$ & \\
\hline-78.13 & -68.04 & -53.49 & -56.04 & محاكاة العطب تمثيلياً باستخدام (FEM) \\
\hline-56.59 & -62.42 & -42.7 & -50.91 & محاكاة العطب عملياً عند ازاحة احد الاقطاب \\
\hline-57.6 & -61.8 & -58.19 & -57.04 & محاكاة العطب عملياً عند استخدام المحامل \\
\hline-53.27 & -49.99 & -33.13 & -35.37 & محاكاة العطب عملياً عند استخدام محامل المعطوبة \\
\hline
\end{tabular}

(1) مقارنة نتائج التشخيص العملية مع نتائج التمثيل لعطب عدم إنتظام الفجوة الهو ائية المختلط.

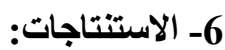

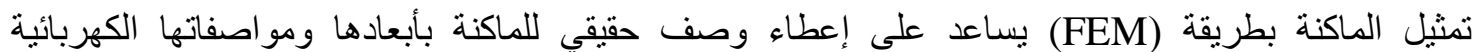

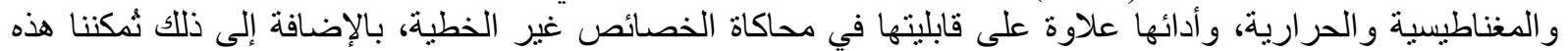

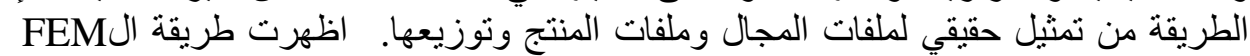

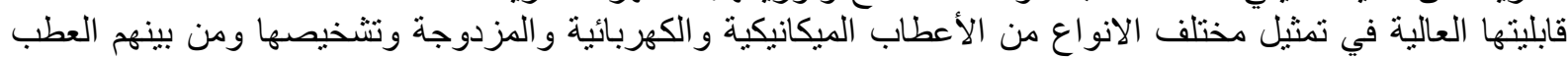

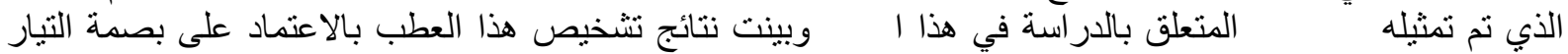

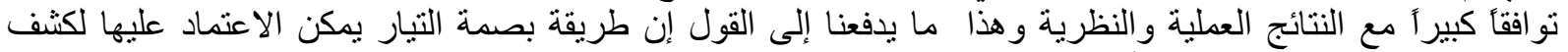

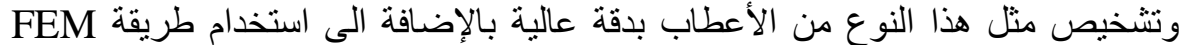

العملية و التي تكون كلفتها عالية اضافة الى صعوبة التية

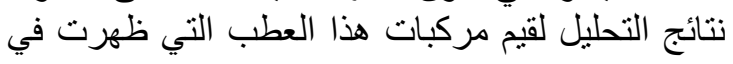

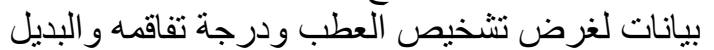
اجر اء الاعطاب على مكائن ذات قدرة عالية. 
الوقت نفسه

$$
\begin{aligned}
& \text { تيار المنتج وتيار المجال، يمكن استتناج إن الماكنة المعرضة } \\
& \text { لهذا العطب } \\
& \text { يعتبر اكثر قساوة من تئن }
\end{aligned}
$$

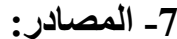

[1] A. Barakat, S. Tnani, G. Champenois and E. Mouni "Analysis of synchronous machine modeling for simulation and industrial applications", Simulation Modelling Practice and Theory Journal, Vol. 18, pp.1382-1396, Oct 2010.

[2] M. Zhang, A. Macdonald, K. Tseng and G.M. Burt, "Magnetic equivalent circuit modeling for interior permanent magnet synchronous machine under eccentricity fault", 48th International Universities Power Engineering Conference (UPEC), Dublin, pp.1-6, Sep 2013.

[3] D. Al-Nimma, M. Matti. "Reactive Power Control of an Alternator with Static Excitation System Connected to a Network", AL Rafiadain Engineering Journal, Vol. 18,No. 3, , Mosul-Iraq, pp. 29-45, Jun 2010.

[4] B. M. Ebrahimi, M. J. Roshtkhari, J. Faiz, S. V. Khatami, "Advanced Eccentricity Fault Recognition in Permanent Magnet Synchronous Motors Using Stator Current Signature Analysis", IEEE Transaction on Industrial Electronics, Vol.61,NO. 4, APRIL 2014.

[5] Basil, M. Saied, Yaser M. Ameen, Mohammed H. Saoud, "Detection of Broken Bars of a Three Phase Induction Motor". Al-Rafiadain Engineering Journal,Vol.20, No. 4 , Mosul-Iraq, pp. 25-32, October 2012.

[6] Basil, M. Saied, Yaser M. Ameen, "Intelligent Technique for Three-Phase Induction Motor Inter Turn Short Circuit Faults Diagnosis Based on Voltage and Current Unbalance Percentage", LAICEEE, Oct., 23, 2010.

[7] M. Akar and . Çankaya, "Diagnosis of Static Eccentricity Fault in Permanent Magnet Synchronous Motor by On-Line Monitoring of Motor Current and Voltage ", Istanbul University-Journal of Electrical \& Electronics Engineering, Vol. 9, pp. 959-967, 2009.

[8] John S.Hsu, Jan Stein "Shaft Signals of a Salient-Pole Synchronous Machine For Eccentricity and Short Field-Coil Detections", IEEE Transaction on Energy Conversion, Vol. 9, pp. 572-578, Set 1994.

[9] I. Tabatabaei, J. Faiz, H. Lesani, and M. T. Nabavi-Razavi, "Modeling and Simulation of a Salient-Pole Synchronous Generator With Dynamic Eccentricity Using Modified Winding Function Theory", IEEE Transaction on Magnetics. Vol. 40, pp. 1550-1555, May 2004.

[10] M. Kiani, W-J. Lee, R. Kenarangui, and B. Fahimi, "Detection of Rotor Faults in Synchronous Generators", IEEE International Symposium Conference on Diagnostics for Electric Machines, Power Electronics and Drives, Cracow, pp. 266-271, Sep 2007.

[11] C. Bruzzese, E. Santini, V. Benucci and A. Millerani, "Model-based eccentricity diagnosis for a ship brushless-generator exploiting the Machine Voltage Signature Analysis (MVSA) ", IEEE International Symposium Conference on Diagnostics for Electric Machines, Power Electronics and Drives, Cargese, pp. 1-7, Sep 2009.

[12] B. A. T. Iamamura, Y. Le Menach, A. Tounzi, N. Sadowski, E. Guillot, T. Jacq and J. Langlet, "Study of Synchronous Generator Static Eccentricities - FEM Results and Measurements", XXth International Conference on Electrical Machines (ICEM), Marseille, pp. 1829-1835, Sept 2012. 
[13] S. Nandi, H. A. Toliyat and X. Li, "Condition Monitoring and Fault Diagnosis of Electrical Motors- A Review”, IEEE Transaction on Energy Conversion, Vol. 20, pp. 719-729, Dec 2005.

[14] I. T. Chelvan, "Non-Invasive Detection of Air Gap Eccentricity in Synchronous Machines Using Current Signature Analysis", Ph.D Thesis, University of Victoria, 2012.

[15] H. Akbari, "An improved analytical model for salient pole synchronous machines under general eccentricity fault", Progress In Electromagnetics Research B Journal, Vol. 49, pp. 389-409, 2013.

[16] K. Hameyer and R. Belmans, "Numerical Modeling and Design of Electrical Machines and Devices". Southampton, U.K.: WIT Press, 1999.

[17] Basil M. Saied, Ahmed J. Ali, “Fault Prediction of Deep Bar Cage Rotor Induction Motor Based on FEM”, Progress In Electromagnetics Research B, Vol. 53, 291-314, 2013, ww.jpier.org/PIERB/pier.php?.

[18] Basil M. Saied, Ahmed . H. Ahmed, "Modeling and Simulation of Salient Pole Synchronous Machine With Comparison Between Actual and d-q Models", Second Scientific Engineering Conference, Mosul, Vol. 2, pp.41-56, Nov 2013.

$$
\text { الملحق (أ) }
$$

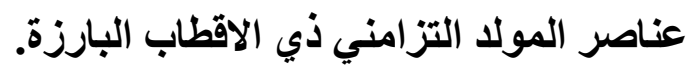

The parameter specification of synchronous generator under study.

\begin{tabular}{|c|c|}
\hline Generator & Data \\
\hline Rated power & $6.2 \mathrm{kVA}$ \\
\hline Rated speed & $1500 \mathrm{Rpm}$ \\
\hline Line voltage & $380 \mathrm{~V} \mathrm{Y}$ \\
\hline Field current & $3.6 \mathrm{~A}$ \\
\hline Number of poles & 4 \\
\hline Outer diameter of the stator core & $254 \mathrm{~mm}$ \\
\hline Inner diameter of the stator core & $177 \mathrm{~mm}$ \\
\hline Length of the stator core & $105 \mathrm{~mm}$ \\
\hline Inner diameter of the rotor core & $53 \mathrm{~mm}$ \\
\hline Number of slots of the stator & 36 \\
\hline Number of conductor per slots & 14 \\
\hline Coil pitch of the stator winding & 7 \\
\hline Number of conductor per pole of the rotor & 420 \\
\hline Pole shoe width & $93 \mathrm{~mm}$ \\
\hline Pole shoe height & $16 \mathrm{~mm}$ \\
\hline Pole body width & $42 \mathrm{~mm}$ \\
\hline Rotor Inertia & $0.0737023\left(\mathrm{~kg} . \mathrm{m}^{\wedge} 2\right)$ \\
\hline
\end{tabular}

$$
\text { تم اجراء البحث في كلية ألهندة = جامعة ألموصل }
$$

